\title{
Coenzyme-Q10 deficiency and stress oxidative in children with autism spectrum disorders.
}

\author{
Elham Mousavinejad*, Mohammad Ali Ghaffari, Seyedeh-Arefe Payami, Nasrin Lamuchi-Deli, \\ Damoon Ashtary-Larky \\ Department of Biochemistry, School of Medical Sciences, Ahvaz Jundishapur University of Medical Sciences, Ahvaz, Iran
}

\begin{abstract}
We assume that serum concentration of total CoQ10 and stress oxidative factors could be used as important biomarkers of therapy. Sixty children (aged 3-12 years), one group consisted of children with autism $(n=30)$ and other consisted of health children $(n=30)$. Children with autism according to the DSM-IV criteria and using CARS were included in the study. All the subjects were Iranian, born and living in the state Khuzestan. This was an original study. The present study

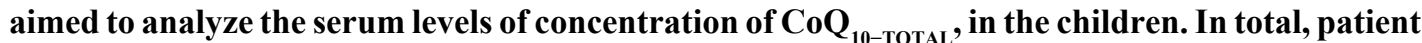
group and health group, including boys and girls, were matched for age, gender, and body mass index (BMI). Serum levels of CoQ10-TOTAL in children with ASDs were significantly lower than that in the healthy children. We propose that serum concentration of $\mathrm{CoQ}_{10 \text {-TOTAL }}$ could be used as relevant biomarkers of CoQ10 supportive therapy. Overall supplementation with Co-Q1 provide promising alternatives to current therapies for neurodevelopmental disorders. CoQ10 is a naturally occurring flavonoid with potent antioxidant, properties that are found in green plants. This study with a larger number of patients is confirmed this previous research.
\end{abstract}

Keywords: O-enzyme Q10, Oxidative stress, Autism spectrum, Disorder, Antioxidant.

Accepted on December 14, 2017

\section{Introduction}

Autistic Spectrum Disorders (ASDs) are the neurodevelopmental disorder resulting by pervasive defects in social interaction and communication, restricted interests and repetitive behaviors [1]. The etiology of ASDs stems from the genetic, environmental and neurological elements [2]. The studies suggested that ASDs may result from an interaction between immunological factors and environmental, genetic with oxidative stress as a mechanism linking these risk factors [3].

The brain is particularly vulnerable to the oxidant threat because of its high rate of aerobic metabolism and readily available oxidizable molecules [4]. Multiple biological processes are related to neurodegenerative and neurodevelopmental diseases such as reduction or inadequate synthesis of oxidative stress and abnormal ubiquitination [5]. Oxidative stress is famous to play a role in some neurological conditions such as Dementia's disease [6,7], bipolar disorder [8] and schizophrenia [9]. Under normal situations, a dynamic equilibrium is between the antioxidant capacity of the cell and the production of reactive oxygen species [10]. Oxidative stress controls the activities of receptor and no receptor types of protein tyrosine kinases, transcription factors such as NF- $\kappa \beta$ and protein kinase $C$ [11]. NF- $\kappa \beta$ that is originating to be dysregulated in ASDs [12]. Multiple molecular features could be observed for the neurodevelopment in the brain of ASDs including oxidative stress and proinflammatory cytokines [13]. Consistent with reduced endogenous antioxidant capacity cause increased oxidative stress biomarkers in children with ASDs [14]. Increased ROS metabolism induced by dysfunctional mitochondria could cause chronic oxidative stress [15].
Coenzyme Q10 (CoQ10) was extra to support mitochondrial role [16]. Coenzyme- Q10 (also called ubiquinone) is a lipid-soluble benzoquinone [17]. CoQ10 is recognized as an intracellular antioxidant that protects mitochondrial membrane protein, membrane phospholipids, and low-density lipoprotein from free radical-induced oxidative damage [18,19]. Psychiatric medication CoQ10 was added to support mitochondrial function [20-22]. The present study has assayed the serum CoQ10 concentration in ASDs children that may contribute to recognize of some individuals to ASDs, especially Iranian.

\section{Materials and Methods}

\section{Study design and participants}

This study was designed as a randomized, parallel, placebocontrolled study. The sample size was calculated using of with $\alpha=0.05$. Thirty children (mean age $7.72 \pm 2.65$; range $3-12$ y) suffering from mild to severe autism spectrum disorders were enrolled into the study, ASDs was diagnosed according to the Diagnostic and Statistical Manual of Mental Disorders, Fourth Edition, Text Revision (DSM-IVTR) American Psychiatric Association [23], the Autism Diagnostic Observation Schedule (ADOS) [24] and using CARS (Childhood Autism Rating Scale, a test combining parent reports and direct observation by the professional) [25]. This study excluded children who had a history of Fragile X disorder, tuberous sclerosis, phenylketonuria (PKU), Lesch-Nyhan syndrome, fetal alcohol syndrome, or history of maternal illicit drug use [26]. In addition, this study excluded any children who had a history of chelation therapy and vitamin or mineral supplements. All children with a history of seizure disorders, severe head injury, and psychotic disorder and any other major acute or chronic physical or mental illnesses 
Citation: Mousavinejad E, Ghaffari MA, Payami SA, et al. Coenzyme-Q10 deficiency and stress oxidative in children with autism spectrum disorders. J Neurol Neurorehabil Res. 2017;2(2):25-29.

were excluded from the control group. After overnight fasting, venous blood was drawn from the antecubital vein, at study enrollment. Participants were group-adjusted for age, sex and body mass index (BMI) (Table 1). All the subjects were Iranian, born and living in the state Khuzestan.

\section{Anthropometric measurements}

All the participants were measured in weight; height and their Body Mass Index were calculated. Body mass index (BMI) (body weight $(\mathrm{kg}) /$ height squared $\left(\mathrm{m}^{-2}\right)$, and standard deviation (SD) score for BMI (BMI-SDS) were calculated (Table 1) [27].

\section{Blood samples}

The fasting blood samples were collected in the morning between 8:00 AM and 10:00 AM. The serum was separated by centrifugation at $1300 \mathrm{~g}$ for $10 \mathrm{~min}$ at $+4^{\circ} \mathrm{C}$ and divided into $150 \mu \mathrm{l}$ of aliquots immediately stored at $-20^{\circ} \mathrm{C}$ where after serum was collected quickly frozen and kept at $-20^{\circ} \mathrm{C}$ in the Medical School laboratory, Jundishapour University of Medical Sciences, Ahvaz, Iran, until analyzed time. The storage time was less than one month.

\section{Biochemical analyses}

\section{Determination of the CoQ10 concentration:}

CoQ10 serum was measured by ELISA kit (My Bio Source).

The biochemical analyses:

Intra-assay Precision (Precision within an assay): $\mathrm{CV} \%<8 \%$.

Inter-assay Precision (Precision between assays): $\mathrm{CV} \%<10 \%$.

\section{Ethics approval and consent}

The study was approved by the regional Ethics Committee of the Medical University of Medical Sciences, Ahvaz, Iran. Written informed consent was obtained from children's parents.

\section{Statistical Analysis}

The sample size was calculated using $\alpha$ of 0.05 and a power of analysis $80 \%$. Data analysis was performed using SPSS (version 19). The values are expressed as mean \pm S.D. (Standard Deviation) Normal distribution and homogeneity of variance of the data were tested by the Kolmogorov-Smirnov and Levene test, respectively. For all the statistical tests, a two-tailed $\mathrm{p}$ value $\leq 0.05$ was considered statistically significant and group differences analyzed using Student's t-test. Accuracy of the

Table 1. Demographic and clinical characteristics of ASDs children and healthy children.

\begin{tabular}{|c|c|c|c|c|c|}
\hline \multicolumn{2}{|c|}{ Characteristics } & $\begin{array}{l}\text { Children with } \\
\text { ASDs }(\mathrm{N}=30)\end{array}$ & $\begin{array}{l}\text { Healthy children } \\
\qquad(\mathrm{N}=30)\end{array}$ & t-value & p-value ${ }^{\ddagger}$ \\
\hline \multicolumn{2}{|c|}{ Age (years) } & $7.72(2.65)^{\star}$ & $7.42(2.65)$ & -0.83 & 0.52 \\
\hline \multirow{2}{*}{ Sex $n$} & boys & $20^{\dagger}$ & 20 & & \\
\hline & girls & 10 & 10 & & \\
\hline \multicolumn{2}{|c|}{ Weight $(\mathrm{kg})^{*}$} & $26.52(8.2)^{*}$ & $26.86(8.25)$ & -0.35 & 0.66 \\
\hline \multicolumn{2}{|c|}{ Height (cm) } & $126.37(15.56)$ & $126.66(15.6)$ & -0.34 & 0.73 \\
\hline \multicolumn{2}{|c|}{$\mathrm{BMI}\left(\mathrm{kg} / \mathrm{m}^{2}\right)$} & $16.15(1.38)$ & $16.29(1.38)$ & -0.16 & 0.79 \\
\hline \multicolumn{6}{|c|}{$\begin{array}{l}\text { BMI: Body Mass Index; } \mathrm{N} \text { are numbers. } \\
\text { *Data are mean (standard deviation). } \\
\text { tData are numbers. }\end{array}$} \\
\hline
\end{tabular}

diagnostic CoQ10 tests was assessed via curve analysis of the Receiver Operating Characteristic (ROC).

\section{Results}

\section{The comparison of CoQ10 serum level between ASDs and control groups}

Mean serum level of CoQ10 for all participants is shown in Table 2. Statistical analyses revealed significant differences between the two groups in measured factor. CoQ10 was higher in the ASDs group in comparison with controls (Table 2).

\section{Comparison of receiver operating characteristic (ROC) curves for CoQ10 in ASDs and healthy children}

A ROC analysis was performed to assess the diagnostic value of CoQ10 level for children with ASDs from all examined cases. This figure shows that serum level CoQ10 cannot be used as differentiating biomarkers in children with ASDs (Figure 1).

\section{Discussion}

Our results show that coenzyme Q10 serum reduces significantly in children with ASDs compared to healthy children. Initial assessments of appropriate antioxidants and dietary supplements help in improving reducing oxidative stress to injuries irreversible brain [28,29]. It seems clear from the present results that coenzyme Q10 may be a

Table 2. Case processing summary.

\begin{tabular}{|c|c|c|c|c|}
\hline \multicolumn{5}{|c|}{ Case Processing Summary } \\
\hline \multicolumn{3}{|c|}{ Group $^{a}$} & \multicolumn{2}{|c|}{ Valid N (listwise) } \\
\hline \multicolumn{3}{|c|}{ Positive $^{b}$} & \multicolumn{2}{|c|}{30} \\
\hline \multicolumn{3}{|c|}{ Negative } & \multicolumn{2}{|c|}{30} \\
\hline \multicolumn{5}{|c|}{$\begin{array}{l}\text { Larger values of the test result variable(s) indicate stronger evidence for a } \\
\text { positive actual state. } \\
\text { a. The test result variable(s): Q10 has at least one tie between the positive actua } \\
\text { state group and the negative actual state group. } \\
\text { b. The positive actual state is ASDS }\end{array}$} \\
\hline \multicolumn{5}{|c|}{ Area Under the Curve } \\
\hline \multicolumn{5}{|c|}{ Test Result Variable(s): Q10 } \\
\hline \multirow[t]{2}{*}{ Area } & \multirow[t]{2}{*}{ Std. Error ${ }^{\mathrm{a}}$} & \multirow{2}{*}{$\begin{array}{c}\text { Asymptotic } \\
\text { Sig. }^{\text {b }}\end{array}$} & \multicolumn{2}{|c|}{$\begin{array}{c}\text { Asymptotic } 95 \% \text { Confidence } \\
\text { Interval }\end{array}$} \\
\hline & & & Lower Bound & Upper Bound \\
\hline .131 & 0.051 & 0.000 & .031 & 0.231 \\
\hline
\end{tabular}

\section{CONCENTRATIN Q10}

$$
\text { a } 10 \text { ASDs } n=30 \text { Q10 Health } n=30
$$

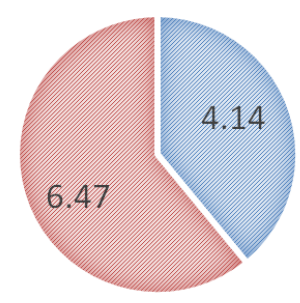

Figure 1. Concentration of serum coenzyme Q10 in ASDs and healthy children. 
protective effect against ASDs, which may be attributed in reducing ROS and inflamation [30]. Coenzyme Q10 supplements can affect in improving activity of antioxidant enzymes [31,32]. The Voltage Dependent Anion Channel is involved in control of ASDs. Therapies, including Co-Q10, have had some achievement on ASDs control [33].

In the present study, the level of serum coenzyme Q10 was low at baseline in our healthy subjects. The serum coenzyme Q10 concentration can be lowered under chelation therapy [34], but we excluded patients who were being treated with chelating agents [35]. As a result, it is believed that supplementation of Co-Q10 in patients with ASDs might improve sustainable antioxidation. An increase in the concentration of Co-Q10 might affect mitochondrial respiratory function [36,37]. CO- Q10 has a better synergistic effect than other antioxidant vitamins such as vitamins $\mathrm{E}, \mathrm{A}$, and $\mathrm{C}$ [38].

The study generally shows some significant irregularities in the biomarkers of oxidative stress in participants diagnosed with ASDs [39-41]. Moreover, a significant inverse correlation is shown between Co-Q10 and ASD severity measured using CARS scoring (Figures 2 and 3). Followed by reducting Co-Q10 in ASDs may be caused by an imbalance between the generation of ROS by endogenous/ exogenous pro-oxidants and the defence mechanism against ROS by antioxidants [42]. Treatment of oxidative stress with antioxidants and other dietary additions may mitochondrial dysfunction ameliorate in individuals with autism spectrum disorders [43]. In addition, it seems clear that coenzyme Q10 has a protective effect against inflammation, which may be ascribed to its antioxidant role [44]. Co-Q10 supportive therapy improved symptoms in children with ASDs, as verbal communication, sleeping, communication with parents, playing games of children, and food rejection [45].

Our study has however three limitations. First, the number of participants was small. Second, the study was designed using just Ahvaz city. larger and extended sample size are needed to enable us to establish the beneficial work in patients with ASDs. Third, our assessment was limited to children. We suggest to it extend the study on adults in various age groups.

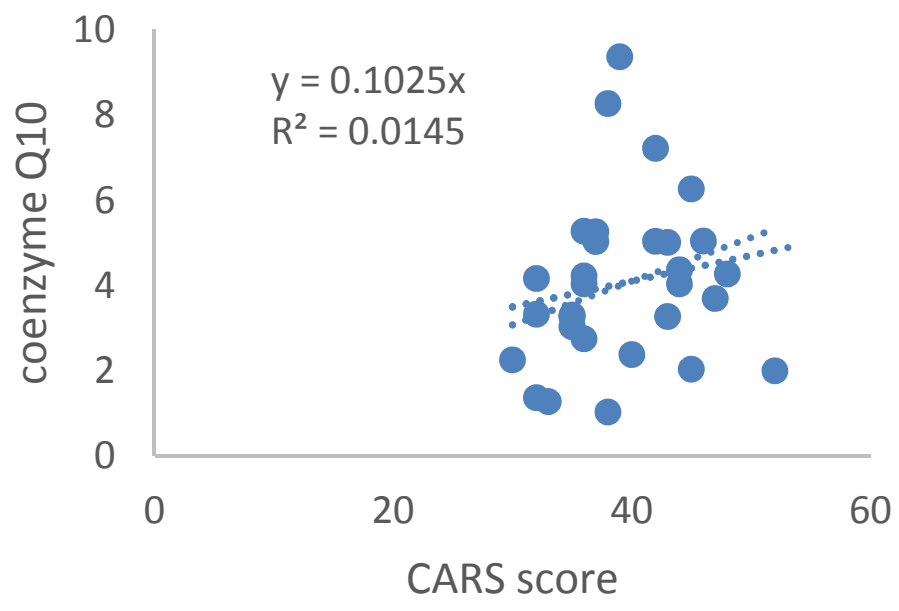

Figure 2. A summary of the correlation between CoQ10 and ASDs severity.
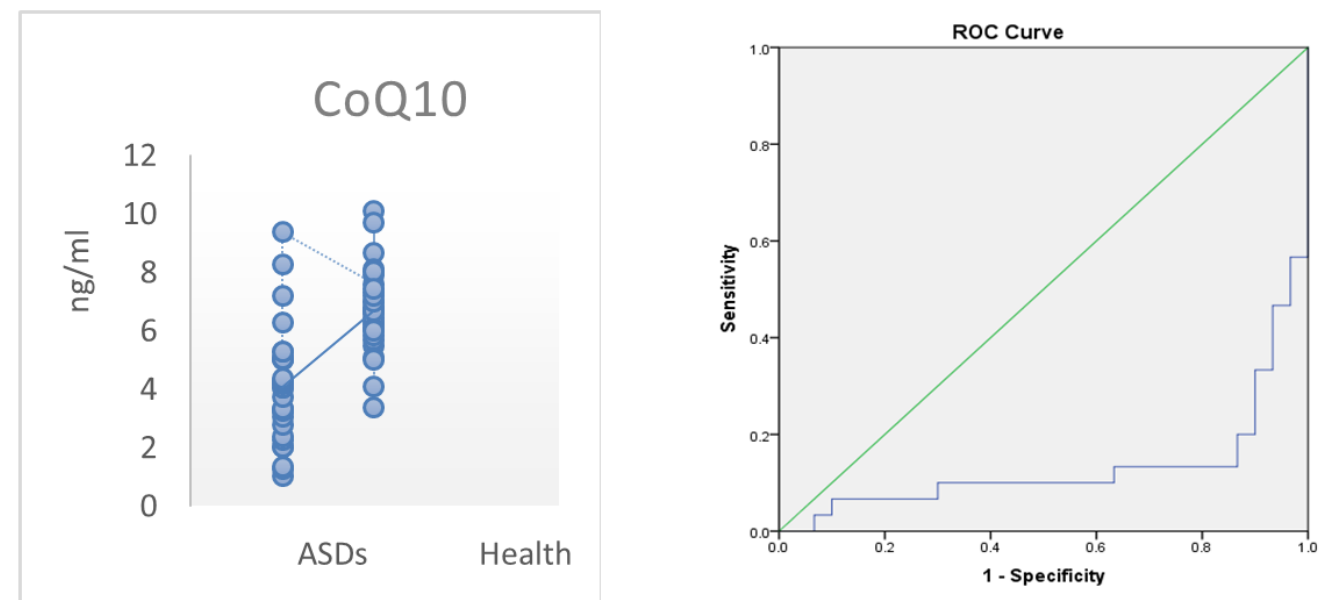

Figure 3. Comparison of receiver operating characteristic (ROC) curves for Co-Q 10 in ASDs and healthy children. 
Citation: Mousavinejad E, Ghaffari MA, Payami SA, et al. Coenzyme-Q10 deficiency and stress oxidative in children with autism spectrum disorders. J Neurol Neurorehabil Res. 2017;2(2):25-29.

\section{Conclusion}

In the future, Co-Q10 could be used as promising therapeutic agents for ASD disorders due to their antioxidative and antiinflammation activities. The apoptosis, necroptosis, and inflammation can lead to the neurodevelopmental disorders such as ASDs, Overall supplementation with Co-Q10 provide promising alternatives to current therapies for apoptosis, necroptosis, and inflammation and also, neurodevelopmental disorders.

\section{Acknowledgment}

This research was funded by a grant from by Cellular and Molecular Research Center (CMRC) at Jundishapur University of Medical Sciences with the following code: CMRC-106. Special thanks to all members of CMRC and families with autistic children for their help and participation in the study.

\section{References}

1. Simonoff E, Pickles A, Charman T, et al. Psychiatric disorders in children with autism spectrum disorders: Prevalence, comorbidity, and associated factors in a population-derived sample. J Am Acad Child Psy. 2008;47:921-9.

2. Mercer L, Creighton S, Holden JJ, et al. Parental perspectives on the causes of an autism spectrum disorder in their children. J Genet Couns. 2006;15:41-50.

3. Chaste P, Leboyer M. Autism risk factors: Genes, environment, and gene-environment interactions. Dialogues Clin Neurosci. 2012;14:281.

4. Bainbridge A, Tachtsidis I, Faulkner SD, et al. Brain mitochondrial oxidative metabolism during and after cerebral hypoxia-ischemia studied by simultaneous phosphorus magnetic-resonance and broadband nearinfrared spectroscopy. Neuroimage. 2014;102:173-83.

5. Chen XJ, Xu H, Cooper HM, et al. Cytoplasmic dynein: A key player in neurodegenerative and neurodevelopmental diseases. Sci China Life Sci. 2014;57:372.

6. Santos JR, Gois AM, Mendonça DM, et al. Nutritional status, oxidative stress and dementia: The role of selenium in Alzheimer's disease. Fron Aging Neurosci. 2014;6:206.

7. Guadagno NA, Moriconi C, Licursi V, et al. Neuroserpin polymers cause oxidative stress in a neuronal model of the dementia FENIB. Neurobiol Dis. 2017;103:32-44.

8. Brown NC, Andreazza AC, Young LT. An updated metaanalysis of oxidative stress markers in bipolar disorder. Psychiat Res. 2014;218:61-8.

9. Hardingham GE, Do KQ. Linking early-life NMDAR hypofunction and oxidative stress in schizophrenia pathogenesis. Nat Rev Neurosci. 2016;17:125-34.

10. Elham M, Mohammad A Ghaffari, et al. Mitochondrial dysfunction in autistic children and oral coenzyme Q10 supplementation treatment. Autism. 2016;6:4.

11. Tobon VCJ, Cuevas E, Torres-Ra M. Receptor for AGEs
(RAGE) as mediator of NF-kB pathway activation in neuroinflammation and oxidative stress. Cns Neurol Disord-Dr. 2014;13:1615-26.

12. Gumpricht E, Rockway S. Can $\omega-3$ fatty acids and tocotrienol-rich vitamin $\mathrm{E}$ reduce symptoms of neurodevelopmental disorders? Nutr. 2014;30:733-8.

13. Ghaffari MA, Mousavinejad E, Riahi F, et al. Increased serum levels of tumor necrosis factor-alpha, resistin, and visfatin in the children with autism spectrum disorders: A case-control study. Neuro Res Inter. 2016;28:2016.

14. Gu F, Chauhan V, Chauhan A. Oxidative stress and mitochondrial dysfunction in ASDs. Autism Res. 2014;9:407-28.

15. Bhat AH, Dar KB, Anees $\mathrm{S}$, et al. Oxidative stress, mitochondrial dysfunction and neurodegenerative diseases: A mechanistic insight. Biomed Pharmacother. 2015;74:101-10.

16. Baruteau J, Hargreaves I, Krywawych S, et al. Successful reversal of propionic acidaemia associated cardiomyopathy: Evidence for low myocardial coenzyme Q 10 status and secondary mitochondrial dysfunction as an underlying pathophysiological mechanism. Mitochondrion. 2014;17:150-6.

17. Sarmiento A, Castro JD, Moran MP, et al. Coenzyme Q10 supplementation and exercise in healthy humans: A systematic review. Curr Drug Metab. 2016;17:345-58.

18. Liu HT, Huang YC, Cheng SB, et al. Effects of coenzyme Q10 supplementation on antioxidant capacity and inflammation in hepatocellular carcinoma patients after surgery: A randomized, placebo-controlled trial. Nutr J. 2016; $15: 85$.

19. Littarru GP, Bruge F, Tiano L. Biochemistry of Coenzyme Q10. Spinger. 2017;23-34.

20. Kaplan BJ, Rucklidge JJ, Romijn A, et al. The emerging field of nutritional mental health: inflammation, the microbiome, oxidative stress, and mitochondrial function. Clin Psychol Sci. 2015;3:964-80.

21. Forester BP, Harper DG, Georgakas J, et al. Antidepressant effects of open label treatment with coenzyme Q10 in geriatric bipolar depression. J Clin Psychopharm. 2015;35:338.

22. Scaini G, Rezin GT, Carvalho AF, et al. Mitochondrial dysfunction in bipolar disorder: Evidence, pathophysiology and translational implications. Neurosci Biobehav R. 2016;68:694-713.

23. Nakao M, Takeuchi T, Fricchione G. Definition of psychosomatic medicine and the applicability of DSM-IVTR to outpatients visiting a Japanese psychosomatic clinic. Psychother Psychosom. 2014;83:120-1.

24. Joshi G, Belser AH, Fitzgerald M, et al. Correspondence of the Massachusetts General Hospital autism spectrum disorder symptom checklist with the autism diagnostic observation schedule. J Am Acad Child Psy. 2017;56:S213-4. 
25. Geier DA, Kern JK, Geier MR. A comparison of the Autism Treatment Evaluation Checklist (ATEC) and the Childhood Autism Rating Scale (CARS) for the quantitative evaluation of autism. J Ment Health Res In. 2013;6:255-67.

26. Kern JK, Geier DA, Geier MR. Evaluation of regression in autism spectrum disorder based on parental reports. North Am J Med Sci. 2014;6(1):41.

27. Wentz E, Björk A, Dahlgren J. Neurodevelopmental disorders are highly over-represented in children with obesity: A cross-sectional study. Obesity. 2017;25:178-84.

28. Rodriguez RA, Jose EGJ, Cabezas FM, et al. Oxidative stress in traumatic brain injury. Curr Med Chem. 2014;21:1201-11.

29. Mahadik SP, Scheffer RE. Oxidative injury and potential use of antioxidants in schizophrenia. Prostag Leukotr Ess. $1996 ; 55: 45-54$

30. Virmani A, Gaetani F, Binienda Z. Effects of metabolic modifiers such as carnitines, coenzyme Q10, and PUFAs against different forms of neurotoxic insults: metabolic inhibitors, MPTP, and methamphetamine. Ann Ny Acad Sci. 2005;1053(1):183-91.

31. Littarru GP, Tiano L. Bioenergetic and antioxidant properties of coenzyme Q10: Recent developments. Mol Biotechnol. 2007;37:31-7.

32. Lee BJ, Huang YC, Chen SJ, et al. Coenzyme Q10 supplementation reduces oxidative stress and increases antioxidant enzyme activity in patients with coronary artery disease. Nutr. 2012;28:250-5.

33. Crane FL, Löw H, Sun I, et al. Plasma membrane coenzyme Q: Evidence for a role in autism. Biol: Targets Ther. 2014;8:199.

34. Cooper JM, Schapira AH. Friedreich's ataxia: Disease mechanisms, antioxidant and Coenzyme Q_ $\{10\}$ therapy. Biofactors. 2003;18:163-71.
35. Weiss KH, Gotthardt DN, Klemm D, et al. Zinc monotherapy is not as effective as chelating agents in treatment of Wilson disease. Gastroenterol. 2011;140:1189-98.

36. Abdin AA, Hamouda HE. Mechanism of the neuroprotective role of coenzyme Q10 with or without L-dopa in rotenoneinduced parkinsonism. Neuropharmacology. 2008;55:1340-6.

37. Fosslien E. Mitochondrial medicine-molecular pathology of defective oxidative phosphorylation. Ann Clin Lab Sci. 2001;31:25-67.

38. Kaur C, Kapoor HC. Antioxidants in fruits and vegetables-the millennium's health. Int J Food Sci Tech. 2001;36:703-25.

39. Shandley K, Austin DW, Bhowmik JL. Are urinary porphyrins a valid diagnostic biomarker of autism spectrum disorder? Autism R. 2014;7:535-42.

40. James SJ, Cutler P, Melnyk S, et al. Metabolic biomarkers of increased oxidative stress and impaired methylation capacity in children with autism. Am J Clin Nutr. 2004;80(6):1611-7.

41. Frustaci A, Neri M, Cesario A, et al. Oxidative stressrelated biomarkers in autism: Systematic review and metaanalyses. Free Radical Bio Med. 2012;52:2128-41.

42. Pham HLA, He H, Pham HC. Free radicals, antioxidants in disease and health. Int J Biomed Sci. 2008;4:89.

43. Rossignol DA, Bradstreet JJ. Evidence of mitochondrial dysfunction in autism and implications for treatment. Am J Am J Biochem Biotech. 2008;4:208-17.

44. Beal MF. Mitochondria, oxidative damage, and inflammation in Parkinson's disease. Ann Ny Acad Sci. 2003;99:120-31.

45. Gvozdjáková A, Kucharská J, Ostatníková D, et al. Ubiquinol improves symptoms in children with autism. Oxid Med Cell Longe. 2014 Feb 23;2014.

\section{*Correspondence to:}

Elham Mousavinejad,

Department of Biochemistry

School of Medical Sciences

University of Medical Sciences

111 Checkerberry Lane

Golestan Blv, Ahvaz

Iran

Tel: 09166151796

Fax: +98-61-33335291

E-mail: mousavinejad.e@ajums.ac.ir 